\title{
Do we still need CRS and HIPEC in colorectal cancer in times of modern chemotherapy and immunotherapy?
}

\author{
Elisabeth Gasser · Pamela Kogler • Andreas Lorenz · Reinhold Kafka-Ritsch • Dietmar Öfner • \\ Alexander Perathoner (iD)
}

Received: 14 June 2020 / Accepted: 18 August 2020 / Published online: 18 September 2020

(C) The Author(s) 2020

\begin{abstract}
Summary Peritoneal carcinomatosis from colorectal cancer is associated with a poor prognosis and is usually treated with systemic chemotherapy and immunotherapy alone. In patients with isolated peritoneal carcinomatosis (PC) without nonperitoneal metastases, however, cytoreductive surgery (CRS) has been shown to significantly improve outcome and to achieve even cure in selected patients in combination with systemic therapy. The additional use of a hyperthermic intraperitoneal chemotherapy (HIPEC) is primarily indicated to control microscopical residual tumor tissue in the peritoneal cavity after successful CRS. Another more recent option is the application of an adjuvant HIPEC to prevent peritoneal carcinomatosis in high risk patients with pT4 cancer or perforated cancer at the time of or after primary surgery. The aim of this short review is to highlight the corresponding available literature and assess the
\end{abstract}

Dr. E. Gasser · PD Dr. P. Kogler · Dr. A. Lorenz • PD Dr. R. Kafka-Ritsch · Univ. Prof. Dr. D. Öfner Assoc. Professor PD Dr. med. univ. A. Perathoner $(\square)$ Department of Visceral, Transplant and Thoracic Surgery, Medical University of Innsbruck, Anichstr. 35, 6020 Innsbruck, Austria alexander.perathoner@i-med.ac.at

Dr. E. Gasser

elisabeth.gasser@i-med.ac.at

PD Dr. P. Kogler

pamela.kogler@i-med.ac.at

Dr. A. Lorenz

andreas.lorenz@i-med.ac.at

PD Dr. R. Kafka-Ritsch

reinhold.kafka-ritsch@tirol-kliniken.at

Univ. Prof. Dr. D. Öfner

dietmar.oefner@i-med.ac.at role of CRS and HIPEC in the context of modern chemotherapy and immunotherapy.

Keywords Hyperthermic intraperitoneal chemotherapy $\cdot$ Cytoreductive surgery $\cdot$ Peritoneal carcinomatosis

In western countries $15-20 \%$ of patients with colorectal cancer present with stage UICC IV disease at the time of diagnosis. Approximately 8-10\% of all colorectal cancer patients present with synchronous peritoneal metastases and up to $40 \%$ develop metachronous peritoneal carcinomatosis (PC) during the course of the disease. Peritoneal metastases are associated with a poor prognosis, a high mortality and a significantly impaired quality of life due to ascites and bowel obstruction [1-4].

The traditional treatment strategy for PC was palliative systemic chemotherapy. A surgical approach was performed only in cases of very limited local involvement, bowel obstruction or perforation; however, according to the development of successful multimodal curative treatment strategies in oligometastatic patients with limited liver or lung metastases, cytoreductive surgery (CRS) and hyperthermic intraperitoneal chemotherapy (HIPEC) in the last two decades have become a legitimate treatment option with curative intent in patients with isolated PC, because it is interpreted rather as a locoregional disease than as a systemic disseminated disease. There is no doubt that surgery is the crucial premise to achieve cure in colorectal cancer. According to this, the application of CRS seems to be logical and essential in PC, even though surgical resection of peritoneal metastases is regarded as an intrinsically incomplete procedure due to the presence of microscopical tumor cells in the peritoneal cavity. To control this residual tumor bur- 
den, CRS was hence associated with chemotherapy. Systemic chemotherapy and targeted agents, however, in different studies did not show the same beneficial effect in peritoneal metastases as in nonperitoneal metastases [5-8]. All these argumentations are indications for the direct use of chemotherapy in the peritoneal cavity in terms of HIPEC in PC.

Different studies showed promising results over historical controls, but until 2003 randomized controlled trials comparing CRS and HIPEC with systemic chemotherapy were missing: Verwaal et al. were the first to detect a randomized controlled survival improvement in patients with peritoneal metastases of colorectal origin after CRS with HIPEC and additional systemic chemotherapy compared to a systematic chemotherapy alone (fluorouracil, leucovorin) [9]. Also, their subsequent study with a minimum followup of 6 years, published in 2008, confirmed a significantly improved survival with a median survival of 12.6 months in the systemic therapy arm and 22.2 months in the HIPEC arm $(p=0.028)$ [10]. Similarly, Elias et al. conducted a randomized trial using systemic oxaliplatin or irinotecan in patients with isolated resectable PC from colorectal cancer and were able to achieve a prolonged median overall survival of 62.7 months after CRS and HIPEC compared to 24 months after systemic chemotherapy alone [11]. Franko et al. 2010 analyzed patients who were treated either with systematic chemotherapy (fluorouracil, irinotecan) alone or with additional CRS and HIPEC. The results showed a significant longer median overall survival in the CRS-HIPEC group (34.7 months vs 16.8 months, $p \leq 0.001$ ), but the CRS-HIPEC group was more likely to receive oxaliplatin and biological agents [12]. In summary, the results of the first studies were clearly in favor of CRS-HIPEC, but from today's perspective an important drawback of all relevant studies is the suboptimal systemic chemotherapy regimen, the heterogeneous systemic chemotherapy regimen in the study groups, and the heterogeneous performance of HIPEC in different centers probably leading to a relevant bias and confounding of the results.

Recently, Quenet et al. presented the highly expected results of the PRODIGE-7 study, a French randomized controlled trial, unfortunately until now published only in abstract form [13]. In this study all patients with colorectal cancer PC received 12 cycles of perioperative systemic chemotherapy consisting of multidrug chemotherapy combinations, including fluorouracil, leucovorin, oxaliplatin (FOLFOX) or fluorouracil, leucovorin, irinotecan (FOLFIRI) with or without biologic agents, such as bevacizumab and cetuximab. Patients were randomized to CRS with or without HIPEC. The results showed an equivalent overall survival rate of 41.3 months and 41.7 months in both treatment arms, but a significantly higher 60 -day major complication rate $(24.1 \%$ vs. $13.6 \%$, $p=0.03$ ) in the HIPEC group. An important limitation of this study, which could have led to a surprisingly high survival in the control group without HIPEC, might be an essential overestimation of the effect size in the trial design. Additionally, it has been speculated that uncertain efficacy of the oxaliplatin-based HIPEC might explain why the study failed to demonstrate a survival difference. Oxaliplatin is considered as one of the standard drugs in locally advanced or metastatic colorectal cancer, but of course not all patients respond to oxaliplatin, especially patients with mucinous histology of the adenocarcinoma, which is common in colorectal cancer PC, have a low response rate after first line therapy. An insufficient duration of the HIPEC ( $30 \mathrm{~min}$ vs. standard $90 \mathrm{~min}$ in most trials) and the use of a nonideal carrier solution (dextrose $5 \%$ ) have been hypothesized, too. Some experts also criticized the unequal inclusion of patients with a high peritoneal carcinomatosis index score $>15$ (30\% in the HIPEC arm and $20 \%$ in the non-HIPEC arm) and the large number of cross-over patients switched from the non-HIPEC arm into the HIPEC arm [14-16].

Another important study was recently published by Baratti et al. [15]. The authors retrospectively compared a prospectively collected group of 96 patients with colorectal cancer and PC treated with an oxaliplatin/irinotecan-based perioperative systemic chemotherapy with targeted agents and CRS, and patients undergoing the same perioperative systemic therapy and CRS plus HIPEC with mitomycin C. The results demonstrated a relatively long median overall survival of 34.8 months (HIPEC group) and 39.3 months (non-HIPEC group). The mitomycin C-based HIPEC in contrast to the oxaliplatin-based HIPEC was not associated with an increased morbidity, but did not have an impact on the prognosis or appear as an independent prognostic predictor; however, both studies, the study of Quenet et al. and the study of Baratti et al. verified the impact of quality of surgery and the potential of modern systemic therapy $[13,15]$. The additional role of HIPEC in the curative treatment of synchronous or metachronous PC in colorectal cancer remains under discussion (e.g. time of exposure, carrier solution, use of chemotherapeutic agents, neoadjuvant or concomitant systemic chemotherapy), and thus, more high-quality studies are needed (e.g. INTERACT trial, CAIRO6 trial) [17-19].

Apart from the standard HIPEC application as curative treatment of synchronous and metachronous PC, another application of HIPEC has emerged in the last years, adjuvant HIPEC in patients with locally advanced colorectal cancer (pT4) or perforated colorectal cancer without visible PC [20-22]. The intention of adjuvant or rather prophylactic HIPEC is to prevent metachronous PC in high-risk patients. Thus, adjuvant HIPEC is performed either simultaneously at the time of primary surgery, or later on as second look or staged procedure after surveillance or adjuvant systemic therapy [22]. The first randomized trials PROPHYLOCHIP and COLOPEC failed to demon- 
strate a significant difference in peritoneal diseasefree survival or overall survival. [20, 21]; however, the amount of available data are rather small to jump to a conclusion and ongoing high-quality trials are still open (e.g. COLOPEC II, PROMENADE and HIPECT4) [22-24]. Recently, pressurized intraperitoneal aerosol chemotherapy (PIPAC) has been proposed as an additional treatment option for patients with PC; however, PIPAC is only palliative and therefore hardly comparable with HIPEC $[6,7]$.

Regarding other tumor forms, data and evidence for CRS and HIPEC appear more complex than in colorectal cancer because of small numbers of patients and missing randomized controlled trials. For example, in PC patients with gastric cancer or malignant peritoneal mesothelioma there is insufficient evidence to recommend CRS and HIPEC outside of clinical trials; however, for patients with ovarian cancer CRS and HIPEC should be considered with at least stable disease after neoadjuvant chemotherapy, if complete cytoreduction is achieved [25].

In conclusion, the therapy of peritoneal carcinomatosis is still challenging for all attending physicians, but multimodal treatment options have significantly improved the outcome of these patients in recent years. In specialized oncological centers the combination of CRS and HIPEC has been shown to be feasible and has gained broad acceptance [26]. Without doubt the quality of surgery represents the essential element to achieve a cure in selected patients with peritoneal carcinomatosis. Thus, cytoreductive surgery is still needed in times of modern chemotherapy and immunotherapy. The role of HIPEC, however. after initial euphoria according to promising results in the first studies, is under discussion, since recent data failed to demonstrate a survival benefit. Highquality studies and randomized controlled trials with adequate patient numbers, comparable treatment concepts and similar inclusion criteria are needed to analyze the additional value of therapeutic or prophylactic HIPEC in colorectal peritoneal carcinomatosis.

\section{Take-home message}

The use of CRS is essential in the treatment of PC in colorectal cancer. The role of additional HIPEC to treat $\mathrm{PC}$ and the role of prophylactic HIPEC to prevent PC is still under discussion.

Funding Open access funding provided by University of Innsbruck and Medical University of Innsbruck.

Conflict of interest E. Gasser, P. Kogler, A. Lorenz, R. KafkaRitsch, D. Öfner, and A. Perathoner declare that they have no competing interests.

Open Access This article is licensed under a Creative Commons Attribution 4.0 International License, which permits use, sharing, adaptation, distribution and reproduction in any medium or format, as long as you give appropriate credit to the original author(s) and the source, provide a link to the Creative Commons licence, and indicate if changes were made. The images or other third party material in this article are included in the article's Creative Commons licence, unless indicated otherwise in a credit line to the material. If material is not included in the article's Creative Commons licence and your intended use is not permitted by statutory regulation or exceeds the permitted use, you will need to obtain permission directly from the copyright holder. To view a copy of this licence, visit http://creativecommons.org/licenses/by/4.0/.

\section{References}

1. Chu DZ, Lang NP, Thompson C, Osteen PK, Westbrook KC. Peritoneal carcinomatosis in nongynecologic malignancy. A prospective study of prognostic factors. Cancer. 1989;63(2):364-7.

2. Segelman J, Granath F, Holm T, Machado M, Mahteme H, Martling A. Incidence, prevalence and risk factors for peritoneal carcinomatosis from colorectal cancer. Br J Surg. 2012;99(5):699-705.

3. Esquivel J, Lowy AM, Markman M, Chua T, Pelz J, Baratti D, et al. The American society of peritoneal surface malignancies (ASPSM) multiinstitution evaluation of the peritoneal surface disease severity score (PSDSS) in 1,013 patients with colorectalcancerwith peritoneal Carcinomatosis. AnnSurg Oncol. 2014;21(13):4195-201.

4. Franko J, Shi Q, Meyers JP, Maughan TS, Adams RA, Seymour MT, et al. Prognosis of patients with peritoneal metastatic colorectal cancer given systemic therapy: an analysis of individual patient data from prospective randomised trials from the Analysis and Research in Cancers of the Digestive System (ARCAD) database. Lancet Oncol. 2016;17(12):1709-19.

5. Baratti D, Kusamura S, Pietrantonio F, Guaglio M, Niger M, Deraco M. Progress in treatemnts for colorectal cancer peritoneal metastases during the years 2010-2015: a systematic review. Crit Rev Oncol Hematol. 2016;100:209-22.

6. Franko J, Shi Q, Goldman CD, et al. Treatment of colorectal peritoneal carcinomatosis with systemic chemotherapy: a pooled analysis of north central cancer treatment group phase III trials N9741 and N9841. J Clin Oncol. 2012;30:263-7.

7. Klaver YL, Simkens LH, Lemmens VE, et al. Outcomes of colorectal cancer patients with peritoneal carcinomatosis treated with chemotherapy with and without targeted therapy. Eur J Surg Oncol. 2012;38:617-23.

8. Gonzalez-Moreno S, Gonzalez-Bayon LA, Ortega-Perez G. Hyperthermic intraperitoneal chemotherapy: rationale and technique. World J Gastrointest Oncol. 2010;2:68-75.

9. Verwaal VJ, van Ruth S, de Bree E, van Sloothen GW, van Tinteren H, Boot H, etal. Randomized trial of cytoreduction and hyperthermic intraperitoneal chemotherapy versus systemic chemotherapy and palliative surgery in patients with peritoneal carcinomatosis of colorectal cancer. J Clin Oncol. 2003;21(20):3737-43.

10. Verwaal VJ, Bruin S, Boot H, van Slooten G, van Tinteren H. 8-yearfollow-up of randomized trial: cytoreduction and hyperthermic intraperitoneal chemotherapy versus systemic chemotherapy in patients with peritoneal carcinomatosis of colorectal cancer. Ann Surg Oncol. 2008;15(9):2426-32.

11. Elias D, Lefevre JH, Chevalier J, Brouquet A, Marchal F, Classe JM, et al. Complete cytoreductive surgery plus intraperitoneal chemohyperthermia with oxaliplatin for peritoneal carcinomatosis of colorectal origin. JClin Oncol. 2009;27(5):681-5.

12. Franko J, Ibrahim Z, Gusani NJ, Holtzman MP, Bartlett DL, Zeh HJ 3rd. Cytoreductive surgery and hyperthermic in- 
traperitoneal chemoperfusion versus systemic chemotherapy alone for colorectal peritoneal carcinomatosis. Cancer. 2010;116(16):3756-62.

13. Quenet F, Elias D, Roca L, Goere D, Ghouti L, Pocard M, et al. A UNICANCER phase III trial of hyperthermic intra-peritoneal chemotherapy (HIPEC) for colorectal peritoneal carcinomatosis (PC): PRODIGE 7. J Clin Oncol. 2018;36(suppl):LBA3503.

14. Ceelen W. HIPEC with oxaliplatin for colorectal peritoneal metastasis: The end of the road? Eur J Surg Oncol. 2019;45(3):400-2.

15. Baratti D, Kusamura S, Azmi N, Guaglio M, Montenovo M, Deraco M. Colorectal peritoneal metastases treated by perioerative systemic chemotherapy and cytoreductive surgery with or without mitomycin C based HIPEC: a comparative study using the peritoneal surface disease severity score (PSDSS). Ann Surg Oncol. 2020;27:98-106.

16. CatalanoV,LoupakisF, GrazianoF, TorresiU, etal. Mucinous histology predicts for poor responserateand overall survival of patients with colorectal cancer and treated with first-line oxaliplatin- and/or irinotecan-based chemotherapy. Br J Cancer. 2009;100(6):881-7.

17. de Boer NL, Brandt-Kerkhof ARM, Madsen EVE, Diepeveen M, van Meerten E, van Eerden RAG, et al. Concomitant intraperitoneal and systemic chemotherapy for extensive peritoneal metastases of colorectal origin: protocol of the multicentre, open-label, phase I, dose-escalation INTERACT trial. BMJ Open. 2019;9(12):e34508.

18. Beal EW, Suarez-Kelly LP, Kimbrough CW, Johnston FM, Greer J, AbbottDE, et al. Impact of neoadjuvant chemotherapy on the outcomes of cytoreductive surgery and hyperthermic intraperitoneal chemotherapy for colorectal peritoneal metastases: a multi-institutional retrospective review. JClin Med. 2020;9(3):748.

19. Rovers KP, Bakkers C, Simkens G, Burger JWA, Nienhuijs SW, Creemers GM, et al. Perioperative systemic therapy and cytoreductive surgery with HIPEC versus upfront cytoreductive surgery with HIPEC alone for isolated resectable colorectal peritoneal metastases: protocol of a multicentre, open-label, parallel-group, phase II-III, randomised, superiority study (CAIRO6). BMCCancer. 2019;19(1):390.

20. Goéré D, Glehen O, Quenet F, Guilloit JM, Bereder JM, Lorimier G, Thibaudeau E, Ghouti L, Pinto A, Tuech JJ, Kianmanesh R, Carretier M, Marchal F, Arvieux C, Brigand C, Meeus P, Rat P, Durand-Fontanier S, Mariani P, Lakkis Z, Loi V, Pirro N, Sabbagh C, Texier M, Elias D; BIG-RENAPE group. Second-look surgery plus hyperthermic intraperitoneal chemotherapyversus surveillance in patients athigh risk of developing colorectal peritoneal metastases (PRO-
PHYLOCHIP-PRODIGE 15): a randomised, phase 3 study. Lancet Oncol. 2020 Sep;21(9):1147-54. https://doi.org/10. 1016/S1470-2045(20)30322.

21. Klaver CEL, Wisselink DD, Punt CJA, Snaebjornsson P, Crezee J, Aalbers AGJ, et al. Adjuvant hyperthermic intraperitoneal chemotherapy in patients with locally advanced colon cancer (COLOPEC): a multicentre, openlabel, randomised trial. Lancet Gastroenterol Hepatol. 2019;4(10):761-70.

22. Baratti D, Sammartino P, Kusamura S, Deraco M, Peritoneal Surface Malignancy Onco-team of the Italian Society of SurgicalO, Ansaloni L, et al. Past, present and future of adjuvant HIPEC in patients at high risk for colorectal peritoneal metastases. Eur J Surg Oncol. 2020 May;46(5):737-9.

23. Arjona-Sanchez A, Barrios P, Boldo-Roda E, Camps B, Carrasco-Campos J, Martin CV, et al. HIPECT4: multicentre, randomized clinical trial to evaluate safety and efficacy of hyperthermic intra-peritoneal chemotherapy (HIPEC) with Mitomycin $\mathrm{C}$ used during surgery for treatment of locally advanced colorectal carcinoma. BMC Cancer. 2018;18(1):183.

24. Bastiaenen VP, Klaver CEL, Kok NFM, et al. Second and third look laparoscopy in pT4 colon cancer patients for early detection of peritoneal metastases; the COLOPEC 2 randomized multicentre trial. BMCCancer. 2019;19:254.

25. Auer R, Sivajohanathan D, Biagi J, Conner J, Kennedy E, May T. Indications for hyperthermic intraperitoneal chemotherapy with cytoreductive surgery: a systematic review. Eur JCancer. 2020;127:76-95.

26. Kogler P, Zitt M, Kafka-Ritsch R, Punter J, Müssigang P, Perathoner A, et al. Cytoreductive surgery (CRS) and hyperthermic Intraperitoneal chemotherapy (HIPEC): a single-center experience in Austria. J Gastrointest Surg. 2018;22(5):884-93.

Publisher's Note Springer Nature remains neutral with regard to jurisdictional claims in published maps and institutional affiliations.

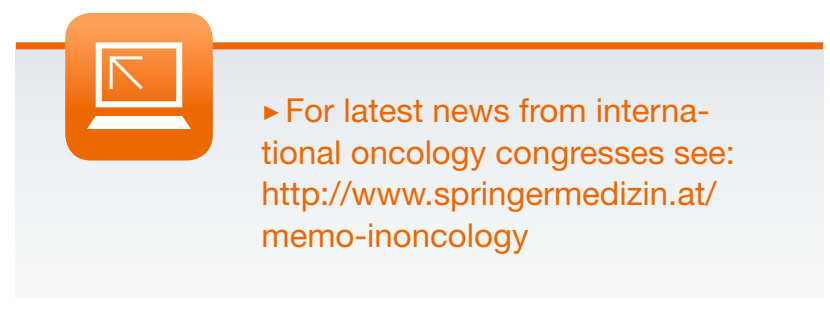

